\title{
Early Design Method to Improve Shading Device Efficiency: The Solar Coupling Index
}

\author{
Jorge Hernán Salazar Trujillo \\ Facultad de Arquitectura,Universidad Nacional de Colombia, Calle 59 A N 63-20 Bl 24, Medellín, C.P.050034, Colombia
}

\begin{abstract}
To reduce solar gains, regions of the sky dome are masked, where the solar path will pass in the moments in which the shade will be desirable. But these regions masked by the shadow devices could also provide diffuse lighting, reducing the need for daytime artificial lighting. When a shadow device is perfectly designed it does not mask more sky than necessary avoiding unwanted solar incursion. During the early stages of architectural design many decisions that will affect the project's energy efficiency have not yet been made and information is lacking to carry out realistic energy or daylighting simulations. The objective was to define an index to qualify, from minimum input data, the effectiveness of a shaded opening by maximizing the visibility to the sky while minimizing undesirable unshaded solar paths. Using basic 3D models and AutoLISP algorithms, it was possible to calculate the aforementioned index. The results demonstrate that similar shadow devices present variable Solar Coupling Indices, explaining why the pre-design of an efficient shadow device is an unintuitive task. The method allows quantifying the gap between the best solar performance achievable and the performance achieved by a specific solar control device, helping to increase the energy efficiency of the architectural enclosures from the early design stages.
\end{abstract}

Key words: Shadow efficiency, shading device, solar control, diffuse lighting, shadow desirability.

\section{Introduction}

The prevention of energy needs caused by unwanted solar incursion tops the list of energy strategies for tropical building construction. Therefore, sustainable construction regulations usually devote their first chapters to issues related to the correct orientation of buildings and the appropriate solar control of its openings. Efficiency in solar control is a prerequisite for the energy quality of a building; in the best interest of time it is important to attend the matter during the early phases of design. Such is a task that is not always undertaken, as seen in the subsequent waste of time and effort that this bad practice causes.

When the methods to achieve high architectural energy efficiency are classified to analyze their effectiveness based on the thermodynamic principle, two categories are created. The first group is defined

Corresponding author: Jorge Hernán Salazar Trujillo, professor, research fields: solar architecture, shading devices, energy efficiency. as the increase in efficiency of the envelope. This involves predominantly balancing the transmission/conduction phenomena, diminishing the surface exposed to solar radiation and specifying materials with a better relationship between their light transmission and solar gain coefficients. In a second group, when the solution is oriented to use only opaque materials, the energy efficiency will depend on the corresponding shape of solar control devices and shade desirability. To emphasize, in the first group the solution would involve thermodynamic modeling whereas, in the second group it would be necessary to optimize geometric form to ensure a form match between a set of opaque objects and certain regions of the sky dome.

Certainly, this classification is precarious because there are heated shadows, semi-darkness, double facades and combined solutions between opaque elements and translucent surfaces. However, it is useful to highlight that the energy optimization of a building belonging to the first group will always 
require the coherence of specialists who carry out the necessary calculations to specify the optical and thermal properties of the translucent materials. In the early phases of design, it is very difficult to achieve, because a high degree of accuracy is required to model natural light based on climate information [1-3]. Additionally, energy simulations require a lot of initial information: thermal properties of materials that have not yet been chosen, structural elements that have not yet been the objective of structural calculation, colors and finishes not yet defined [4, 5]. Unfortunately, when the design team is finally ready to share enough architectural information to do a dynamic simulation of energy performance and lighting blends, the design flexibility is no longer as achievable as it would have been during earlier design phases [6].

A benefit exists in the case of buildings where the solar solution is predominantly dealt with opaque elements: to cast opportune shade in the appropriate areas can be achieved exclusively with a correct size, shape and position, requiring little initial input information. To increase the solar efficiency of a building belonging to the second group means maximizing the relationship between the shape of its openings, the shadow devices and the periods in which the shadow will be desirable. This places the task within the territory of architectural form design. Early decisions have the greatest impact on the future energy performance of a building. If the first materials chosen to start a detailed study of energy efficiency already guarantee that the solar efficiency of each opening is approaching the optimum level, the starting point of the technical teams will help to accurately reach optimal level in a swift and economical way.

\subsection{Solar Coupling}

The purpose of a solar control device is simple: to prevent the visibility towards the sectors of the sky dome through which the solar path will pass during the periods of desirable shade. If a solar control device allows visibility to these sectors, solar paths that should be masked will not be, allowing unwanted solar incursion. As a result, the occupants of the building will begin to close curtains and blinds with the intention of blocking the solar rays that generate overheating and visual discomfort [7]. This situation limits the possibilities of using natural lighting, a reduction of visual contact with the environment, an increase in the requirements of daytime artificial lighting and greater cooling needs, to finally, raise the energy needs of the building. The result will be a higher initial investment in equipment, a greater use of active cooling and notwithstanding, a solar incursion that exacerbates the precarious conditions of visual ergonomics (Fig. 1).
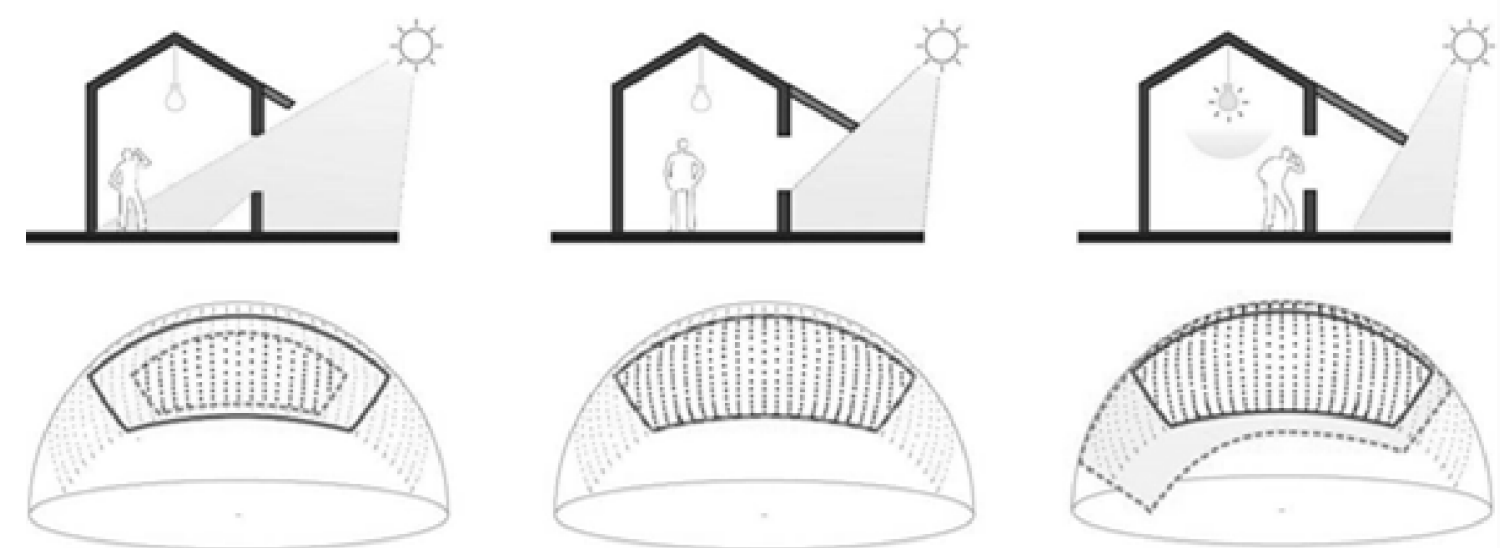

Fig. 1 Established relationships between solar control devices and the possibilities of natural lighting usage. 
On the other hand, if the solar control device masks a larger part of the sky dome than necessary, the opposite will occur and there will be excessive shade. Although there will not be thermal discomfort or exposure to inconvenient solar glare, the use of natural lighting will not be sufficient because the shadow device masks regions where the possibilities to take advantage of diffuse lighting would have been optimal [8]. The result is openings that do not appropriately take advantage of the light benefits that they could have offered. The excess of shade may require reinforcement with daytime artificial lighting and the result will finally be the same: an increase in the energy needs of the building.

Shade excess and deficit are situations to be prevented because both extremes move solar control devices away from their optimum point of operation at the cost of sacrificing energy efficiency and the environmental quality of the building [9]. A point between the two extremes offers the ideal solution, in which the shadow device does not allow the blockage of any undesirable solar rays or unnecessarily masking regions of the sky dome that provide visual contact with the outside, diffuse beneficial lighting and/or, desirable solar radiation. The perfect match between the required shadow and the projected shadow is called Solar Coupling and depends on the geometrical relationships between the opening, the solar control device, and the set of intervals where shadow desirability is optimal within the enclosure. In practice, achieving an ideal Solar Coupling is rare, but having a measure that quantifies the distance of a shadow device from the ideal condition would facilitate the decision-making process during the early stages of architectural design.

\section{Method}

The calculation of the portions of the sky dome masked by a solar control device can be done using shadow masks, a classic solar design tool that allows the assessment of the quality with which a solar control device fulfills its task. Many authors [10-14] include in their books shadow masks for basic architectural forms because superimposing a shadow mask on a solar chart and counting the number of blocked solar positions is the simplest way to estimate their efficiency. On the other hand, counting open solar positions is the simplest way to estimate the optimal margin. But since each shape has its own mask possibilities, it is necessary to apply a simple but rigorous geometric procedure to evaluate design alternatives.

Shadow masks are two-dimensional projections of spherical polygons obtained by contouring any opaque form from a point. For straight prisms with horizontal or vertical edges, the shadow mask is a combination of straight lines segments, ellipses, and arcs that follow each other until a closed figure is isolated. However, in the case of curved elements, inclined planes, and other irregular shapes, it is necessary to trace the masks point by point. Producing shadow masks for complex shapes, including elements of the environment that can restrict solar accessibility, and trying to measure areas with the purpose of comparing the performance of similar solar control devices, are tasks so laborious and imprecise that without computational support they are qualified as unviable.

\subsection{Masking of the Sky Dome}

Any solid object, regardless of its geometric complexity, can be used as a starting point for tracing a shadow mask. Achievable through the application of the steps illustrated in Fig. 2: (a) deconstruct the solid into flat polygons, (b) insert nodes between the vertices that isolate each of the edges to transform each side of the polygons into segments as precise as the level requires, (c) move each node from its original coordinates to a spherical surface with a radius large enough for the parallel effect to become negligible and (d) project the nodes of the spherical polygon obtained on the base plane to define a closed two-dimensional polygon with relation to the shadow mask of the object. 

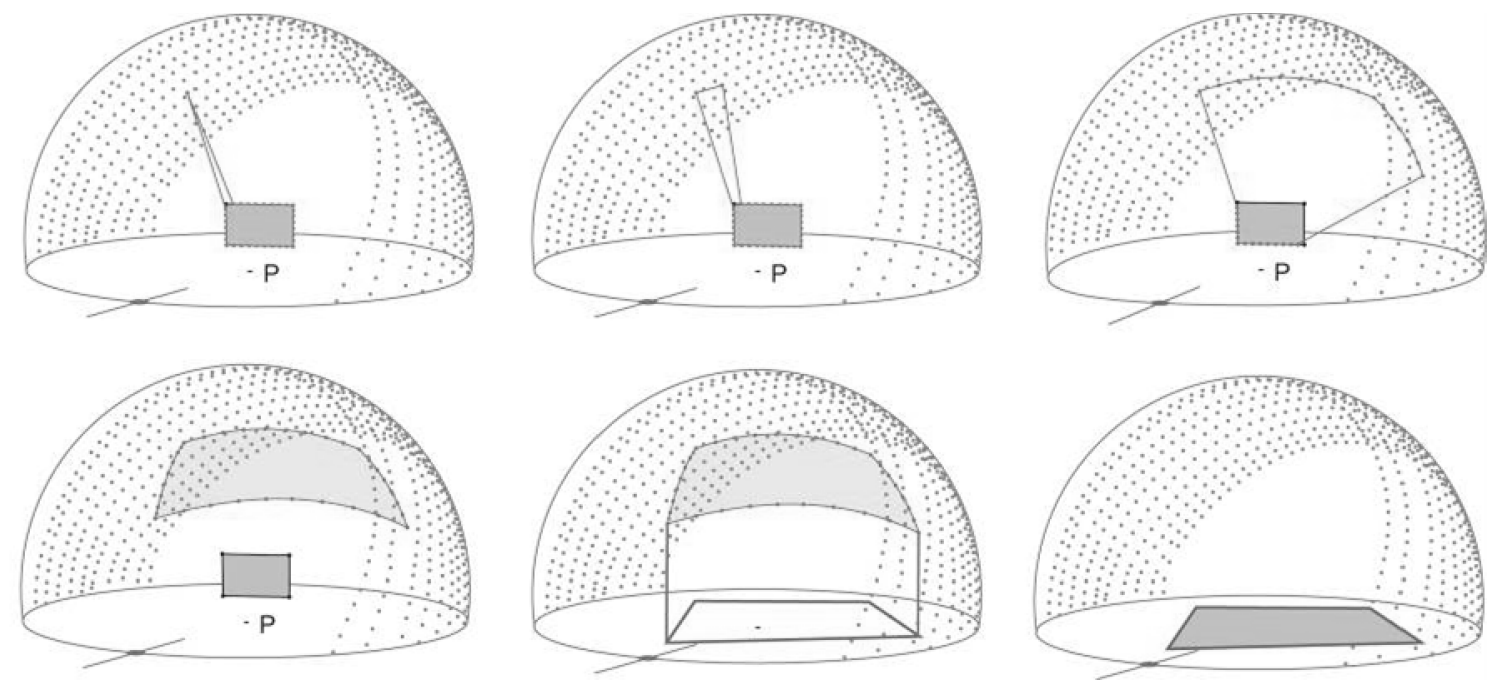

Fig. 2 Shadow mask generation from a flat three-dimensional polygon. This technique allows the transformation of any opaque object into its corresponding spherical masking polygon and then into the required shadow mask.

Regardless of the geometric complexity they have, the calculation of a shadow mask for any type of opening and solar control device is calculated by the application of geometric transformations on individual points instead of deriving the trigonometric functions of complex shapes. In this investigation intermediate nodes were generated by linear interpolation, inserting nodes between vertices, which replace each edge of each polygon into numerous line segments of a maximum of $0.2 \mathrm{~m}$ in length. Then each node was moved to a spherical surface, representing the sky dome, of a radius of $10 \mathrm{~km}$. In respect to the movement of nodes, the laws of triangle symmetry were used from a point that operates as an origin of the polar coordinate system. The Boolean Union of the projection of all these three-dimensional polygons on the horizontal base plane is the mask in question.

\subsection{Shade Desirability}

Shade desirability is defined as the time intervals during which the presence of shade will be desirable. This depends on the schedule/calendar of use of the facility, the climatic characteristics of the site, the distribution of the furniture and the characteristics of the finishes, among other considerations. In order to process this geometric information, it is necessary to translate schedules and calendars in a closed spherical polygon that defines regions of the sky dome that should be masked. Shadow desirability masks are the spherical translation of the shade desirability calendar/schedule. To define these regions Spencer's solar mechanics equations [15] were used to calculate the solar angles for the site under examination, by isolating points on a sphere corresponding to the apparent positions of the solar disk at each date and time of the year. Then, three-dimensional segments were drawn that connect these points until a closed spherical polygon is formed, which includes the periods where the shadow will be desirable. The nodes of this spherical polygon were processed identically to the rest of the shadow masks to project them onto the horizontal base plane and transform them into closed flat polygons.

\subsection{Classification of Regions}

Once projected, the set of shadow masks and shadow desirability masks join and overlap each other defining regions and categorizing them into one of the following four groups: A: the shadow is not desirable, and the region is visible because it was not masked. This corresponds to regions of the sky that can provide diffuse natural lighting without causing discomfort due to unwanted solar incursion. The lighting needs indicate how large it should be. B: the shadow is desirable, but the region is visible because it was not 
masked by any shadow mask. It is the best interest to minimize this group in order to reduce unwanted solar incursion. $\mathrm{C}$ : the shadow is not desirable because the region does not belong to any polygon of shade desirability, but since the region is masked, it will not be able to provide natural lighting even though it has the potential to do so. D: the shadow is desirable because the region belongs to some polygon of desirable shade, but at the same time it is included in at least one shadow mask, so inconvenient sun conditions will not arise.

To calculate the relative area that each of these four groups possesses, a point count was used. 100,000 points were randomly distributed on the surface of the sphere and then projected onto the horizontal plane and processed one by one, determining whether or not they belonged to one or more of the polygons. To determine if a point is included in a closed polygon, a standard algorithm was used to elaborate the computational graphs [16] that had previously been adapted for architectural applications [17]. Classifying each of the points as belonging to one of the four groups, counting them, and converting the sum into percentages is what defines the proportions in which the sky dome is distributed between the opening under examination, the opaque planes that define it, and the shadow devices that shade it.

\subsection{Computation Index of Solar Coupling}

Solar Coupling is a characteristic that depends on the size of the shadow mask and its correct positioning in a way that minimizes Group B (desirable shade in unmasked region) to as small as possible. A perfect coupling would result in a value of $\mathrm{B}$ equal to $0 \%$, a condition unattainable for most openings, unless the shade desirability conditions are very low, and the orientation of the opening is especially favorable. Since the simplest way to obtain a value of $\mathrm{B}=0$ is to not construct any windows, the measurement to assess the efficiency of the solar control devices will be incomplete without a second scale associated with the visibility towards the sky dome. This second figure corresponds to Group A (unwanted shadow in unmasked region), whose value should be as large as the natural lighting needs of the facility require.

The combination of the values obtained for A and B allows the assessment of the effectiveness of solar control offered by any opening along with its accompanying solar control devices. The conditions to achieve the maximum level possible of Solar Coupling are when $\mathrm{B}=0$ and $\mathrm{A}=(1-\mathrm{D})$. They are useful theoretical figures for semi-confined public spaces in which there is some coverage and no enclosures, but inappropriate for any building with vertical enclosures in which openings are implemented, so it is necessary to employ an analysis starting line that operates as a reference of the process and allows comparisons between solar control alternatives (Fig. 3).

$\mathrm{Ax}$ and $\mathrm{Bx}$ being the percentages obtained when evaluating an analysis point, the remaining $(R)$ is defined as the percentage of solar positions that are desirable to have masked but still remain unprotected from direct solar rays in comparison with the base case. Alternatives with a value of $R$ greater than $100 \%$, will display an undesired solar penetration superior to the base case, while values below $100 \%$ will have better solar performance. A value of $R=0 \%$ corresponds to the maximum level of solar control, when all unwanted solar rays are masked.

On the other hand, the percentage of visibility $(V)$ corresponds to the sky dome region free of shadow requirements and visible in comparison with the results obtained for the base case. Values of $V$ above $100 \%$ correspond to solar control devices with better performance because they allow greater visibility to the sky dome compared to the base case. Values below $100 \%$ correspond to openings that reduce the possibilities of using natural light. Minimizing the remaining $(R)$ while maximizing visibility $(V)$ improves the efficiency of the solar control of shadow devices. The Solar Coupling Index is, therefore, defined as an ordered pair of the shape $(R, V)$. 


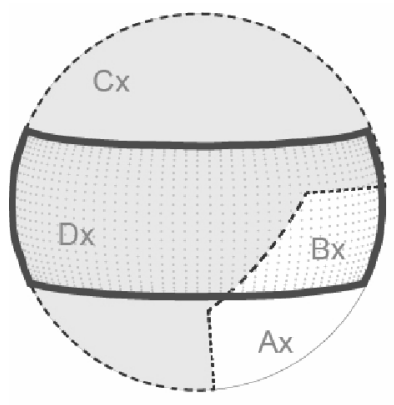

BASE CASE

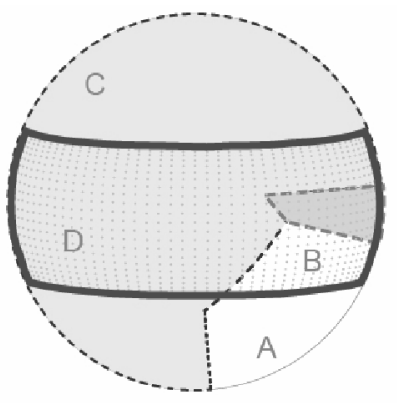

Solar Mask Shade Desirability

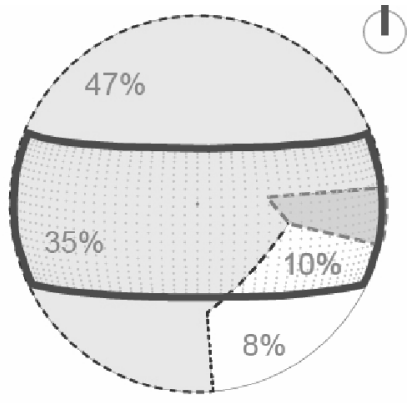

B: Remaining $(R)=B / B x$

A: Visibility $(\mathrm{V})=\mathrm{A} / \mathrm{Ax}$

Fig. 3 Regions in which a studied opening divides the sky dome. Left: masks used as a comparison reference and whose Ax and $\mathrm{Bx}$ results operate as divisors to transform all the results into percentages.

\subsection{Representational Resources}

To represent the relationships between both scales, a scatter diagram was used in which the x-axis corresponds to the remaining $(R)$ values: as a point approaches the origin it will not permit masking a smaller part of the shadow desirability polygon. If the result moves away from the origin, it will be offering a shadow level similar to that of the base case. Where the ordered values of $V$ are located: as a point approaches the origin a greater part of the sky dome will be masked and as it moves away from the origin, it will progressively allow greater visibility towards the sky dome. The base case is located in the upper right part of the diagram, with values of $R$ and $V$ of $100 \%$, as illustrated in Fig. 4. The origin corresponds to the absence of openings: zero unwanted radiation and zero visibility towards the sky dome, while the upper end of the vertical axis corresponds to the best opening possible (zero unwanted radiation and maximum visibility towards the sky dome).

In Solar Coupling diagrams, four cases are presented, as outlined in Fig. 5. Case $1(R<R x)(V<V x)$ and Case $3(R>R x)(V>V x)$ : outline alternatives where there is a tied relationship between undesirable solar radiation and the utilization of the light obtained. Vertically aligned points correspond to alternatives that mask the unwanted light incursion equally, but the one that is higher will have better solar performance because it will offer more light utilization. Alternatives that appear represented as horizontally aligned points will offer such a level of light utilization, but the one on the left will be preferable because it will have less unwanted solar incursion.

The alternatives classified in Case 3 will only be convenient if it is necessary to provide a higher level of illumination than in the base case and between neighboring points, those which, when joined with the origin, define a segment with a greater slope, so that the increase in unwanted sunlight is accompanied by more light input. In Case 1, the lower slope options would be preferable, as the additional shadow would be obtained at the cost of a small reduction in the light contributions that the solar control device allows.

Case $2(R<R x)(V>V x)$ : shadow devices that reduce unwanted solar incursion while raising the visibility to the sky dome, are the ideal quadrant. Any alternative that belongs to this area will be, from the point of view of solar control efficiency, preferable to the base case.

Case $4(R>R x)(V<V x)$ : shading devices that increase the unwanted solar incursion while reducing visibility to the sky dome. It is an unfavorable condition; these are the completely disposable alternatives from the point of view of solar control efficiency. 

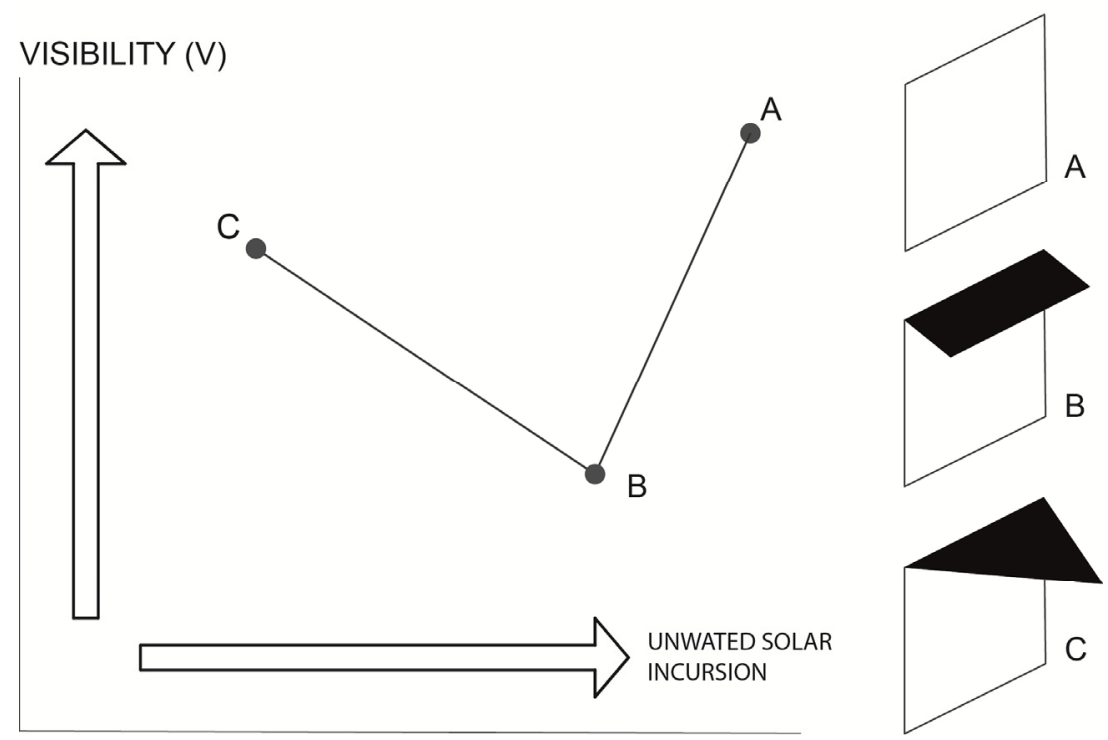

REMAINING (R)

Fig. 4 Relationships between remaining $(R \%)$ and visibility $(V \%)$ of the base case and two solar control alternatives for the same opening. Opening $C$ offers less shade than $B$ and for this reason is on the left, but as it generates less obstruction of the sky dome, it has a greater visibility and for this reason is higher than $B$.

VISIBILITY (V)

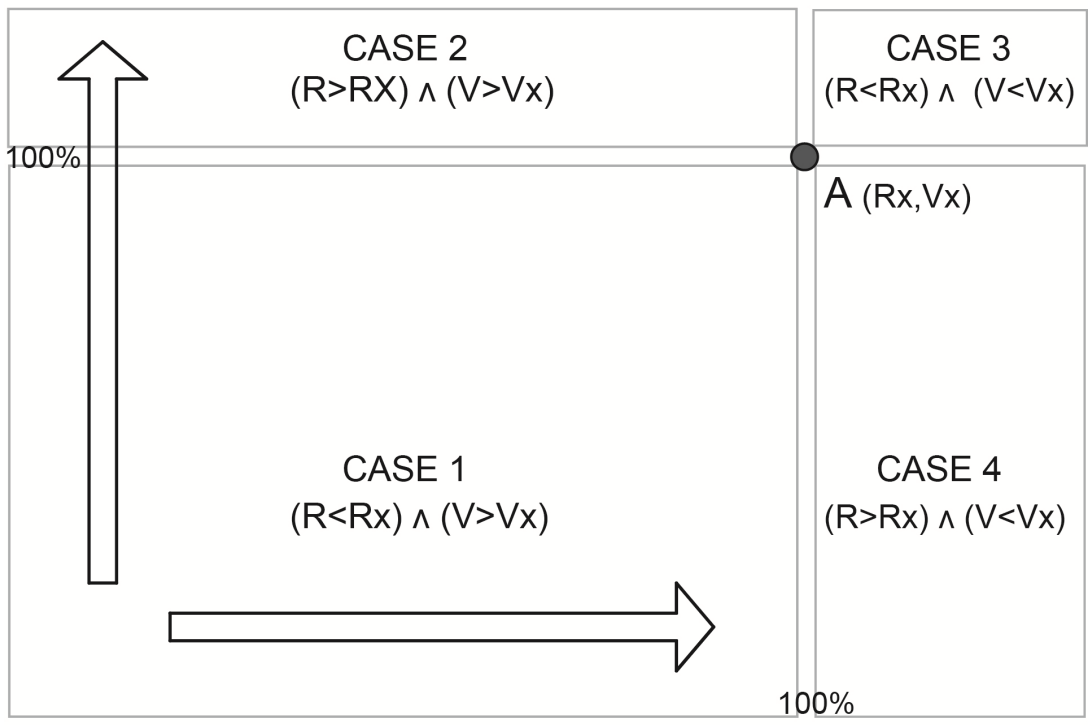

REMAINING (R)

Fig. 5 The four cases that represent the pairings of the remaining and the visibility from the results of $R$ and $V$ obtained when evaluating a base case.

\subsection{Definition of the Analysis Point}

The design of spherical polygons and their projections for obtaining shadow masks are made from a single source and therefore the results obtained are valid for this single point, although they are considered representative for the rest of the opening. This gives a basis to reasonable doubt about the validity of the results. Therefore, the solution that is often applied is to choose a midpoint from the opening (both in length and height) in order to distribute the error equally. This indicates calculating with a point normally very high. 
For example, for an opening with a window sill at 1.0 $\mathrm{m}$ and a window head at $2.20 \mathrm{~m}$, the evaluation point would be located at $1.60 \mathrm{~m}$ above ground level, which may turn out to be a point that is not very representative for most tasks and furniture arrangements.

A second more conservative option is to choose a point that has the least chance of shade, regularly located at floor level if a glass door is evaluated, or at the height of the window sill if it a window is being evaluated. In this way the overall solar performance of the opening will be better than that of the point used to make the analysis. This is an advisable option when energy efficiency is a priority, but an excessive measure if the premise is to ensure conditions of visual ergonomics, in which case the evaluation point could be at eye level of the work plane (if the furniture distribution suggests it) or slightly below the eyes in standard standing position if what is being shaded is a corridor. It is clear that defining the position of the analysis point implies consulting the conditions of which the site will be used and the associated human factors. So, the criteria will vary according to each project, although when performing comparative evaluations of several solar control alternatives it will be necessary to be consistent in the height and the position of the analysis point.
To quantify the impact of choosing between different height options of an analysis point, a test was completed comparing two shadow devices for the same opening. Twenty-four (24) progressively higher points were used, starting at level $0.0 \mathrm{~m}$ and ending at $2.40 \mathrm{~m}$ above the floor. As can be seen in Fig. 6, there are no representative differences to locate the analysis point at $170 \mathrm{~cm}$ above the floor. Both series present linear relationships with a correlation coefficient of 0.98 and the same slope. Therefore, the results that would be obtained by placing the analysis point higher or lower between the interval of $0.0 \mathrm{~m}$ and $1.7 \mathrm{~m}$ will always lead to the same conclusion. That is to say, when using a different point at a different height, a less viable option is impossible to find.

\section{Results and Discussions}

This method was applied to the facades designed at the Milla de Oro Business Center, 65,000 $\mathrm{m}^{2}$ office towers located in Medellin, Colombia $\left(6^{\circ} 25^{\prime} \mathrm{N}, 75^{\circ} 06^{\prime}\right.$ $\mathrm{W}, 1,550$ masl), designed by AIA architects in 2012, Fig. 7. From the early design phase, a team of architects set out to achieve a high energy efficiency to opt for a "Core and Shell" Gold Leed Seal using shadow devices and without resorting to glass surfaces with very high reflection coefficients, a common practice

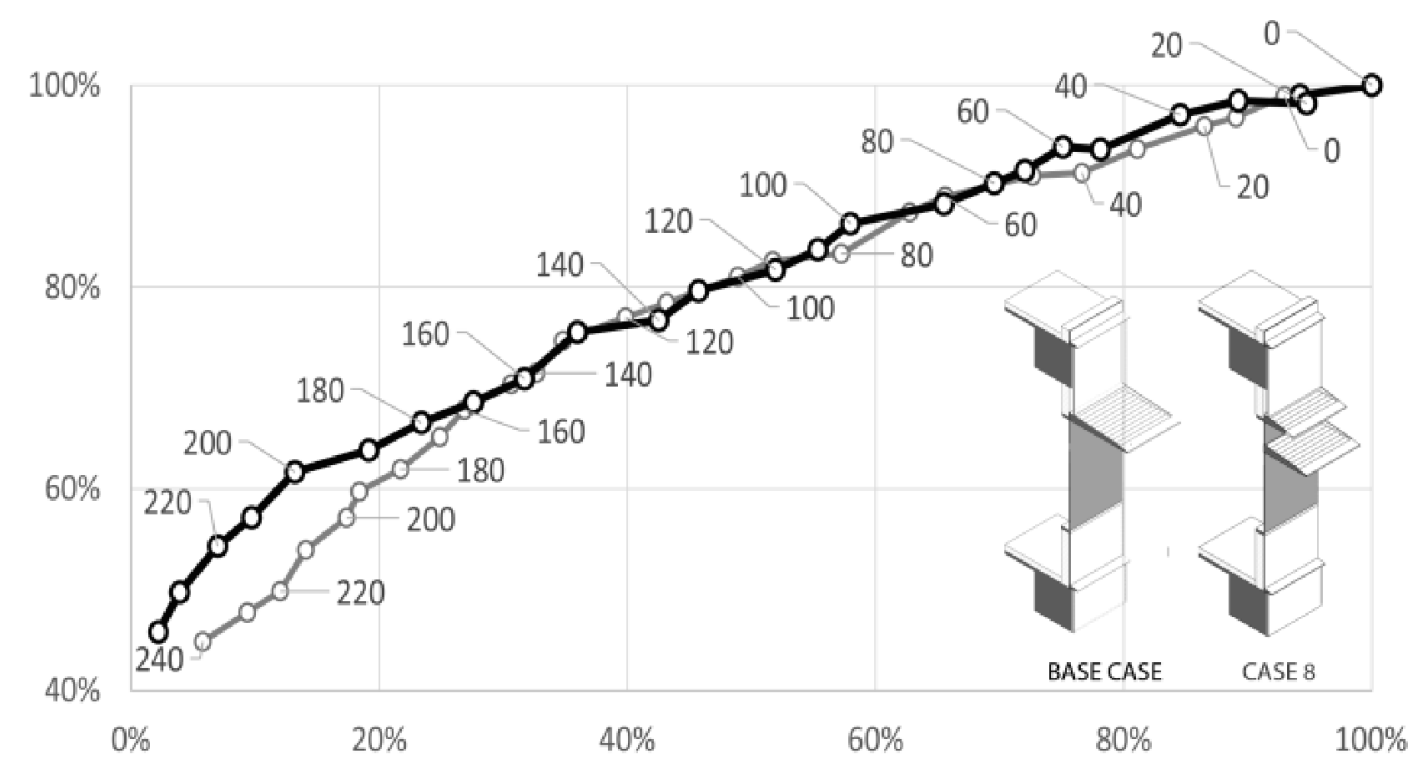

Fig. 6 Two-span Solar Coupling diagram using 24 evaluation points at different heights, starting at floor level and climbing $0.1 \mathrm{~m}$ each time. 

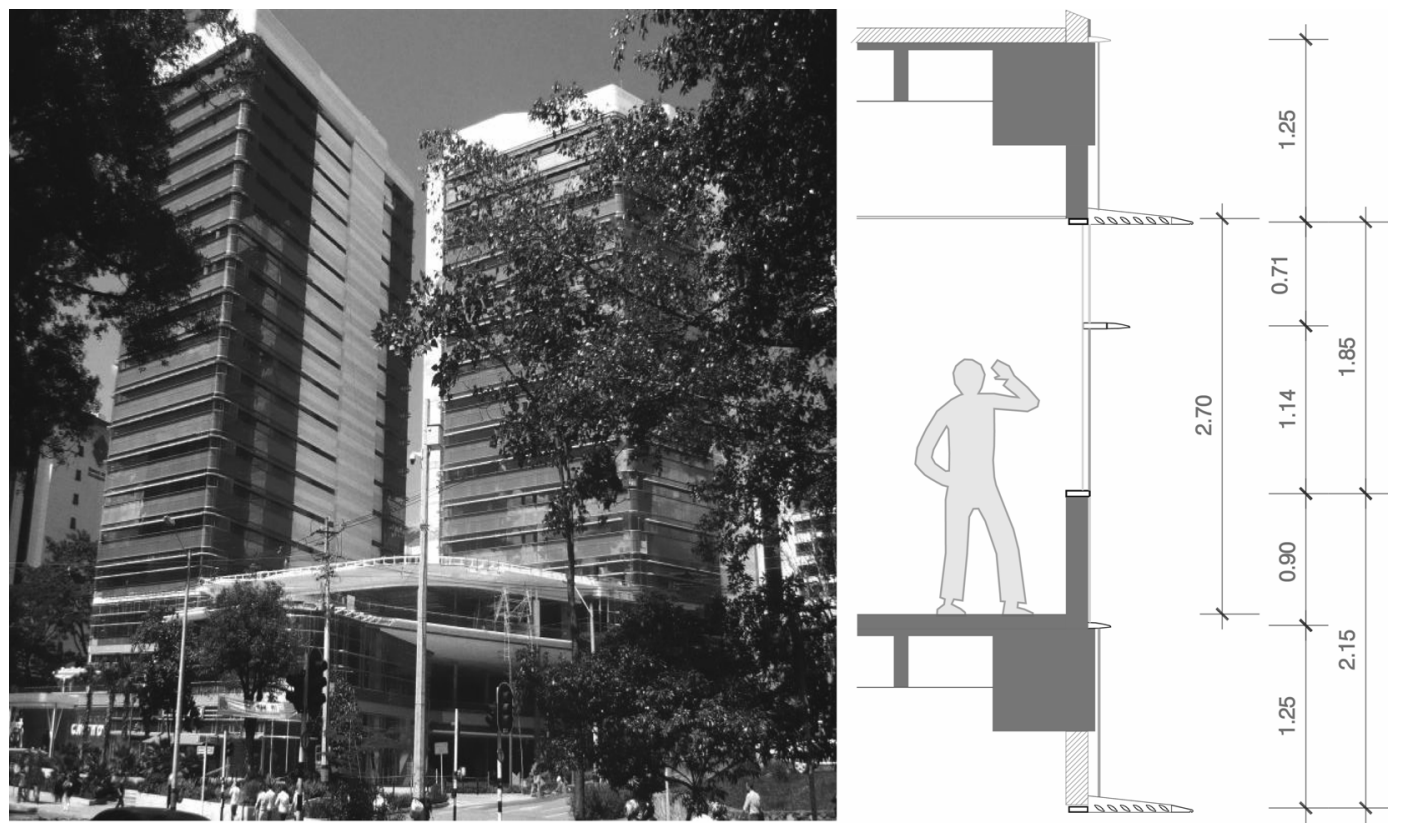

Fig. 7 Milla de Oro Business Center. Medellín, Colombia. On the right, section of the built facade.

of buildings in the city. The starting point of the process to measure and position the solar control devices was in order to achieve a solar performance similar to that offered by continuous eaves of $1.10 \mathrm{~m}$ wide installed at $2.70 \mathrm{~m}$ height. For this reason, these are the characteristics of the base case.

The project has two western facades and their orientations (S50W and $\mathrm{N} 69 \mathrm{~W}$ ) are sufficiently different to merit a differential design. However, these shadow devices have a unique design because the towers have rounded edges. The facades to the northwest (N69W) have the highest levels of solar exposure and the largest glare area, which is why the solar control devices were designed and optimized for this orientation (Fig 7). This explains why the Solar Coupling results of the other orientation demonstrate a solar performance much higher than the base case because it is better protected from the sun.

The Solar Coupling calculations were made for the lowest points of the windows, that is, at the height of the window sill, because the main purpose was to reduce the energy requirements of artificial air conditioning equipment. The shade desirability was defined for working hours and includes an interval between 7:00 and 17:00 hours during every day of the year. To represent the shade desirability, a three-dimensional model was used to perform the calculations based on a case consisting of just 5 plane segments (two sides, one cover, one bottom and one horizontal eaves for a total of 12 three-dimensional coordinates). The following alternatives require between 5 and 7 planes, depending on the number of eave alternatives that could be had in the solar control evaluation process.

All the design alternatives, together with the corresponding material requirements expressed in $\mathrm{m}^{2}$ of aluminum per linear meter of facade per floor, illustrate the explorations made during the early architectural design phase. In the beginning, (alternatives 2-4) the aluminum needs were very high because the possibility of using only clear glass was being evaluated. The corresponding results of the Solar Coupling Index (Fig. 8) allow the comparison of the solar performance of three design alternatives with the same monetary cost.

When the economic investment needed to counteract wind speeds became a consideration of the design variable, it was necessary to reduce the amount of aluminum used. Alternative 5 and the following reveal this reduction. From this point several solar control 


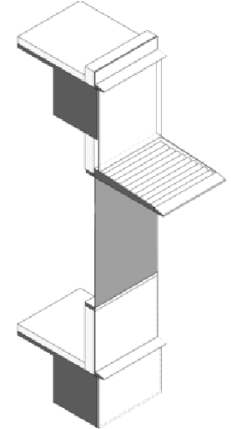

1. $1.10 \mathrm{~m}^{2} / \mathrm{m}$ BASE CASE

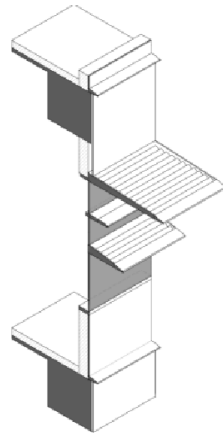

2. $2.05 \mathrm{~m}^{2} / \mathrm{m}$ 3. $2.05 \mathrm{~m}^{2} / \mathrm{m}$ 4. $2.05 \mathrm{~m}^{2} / \mathrm{m}$

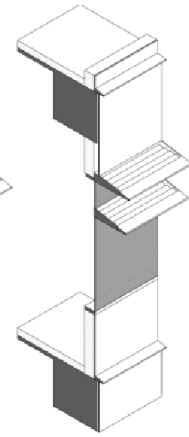

5. $0.95 \mathrm{~m}^{2} / \mathrm{m}$ 6. $1.05 \mathrm{~m}^{2} / \mathrm{m}$ 7. $1.15 \mathrm{~m}^{2} / \mathrm{m}$

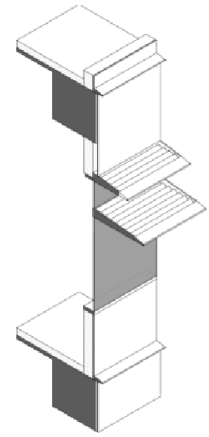

8. $1.25 \mathrm{~m}^{2} / \mathrm{m}$ 9. $1.35 \mathrm{~m}^{2} / \mathrm{m}$ 10. $1.45 \mathrm{~m}^{2} / \mathrm{m}$ 11. $1.55 \mathrm{~m}^{2} / \mathrm{m}$

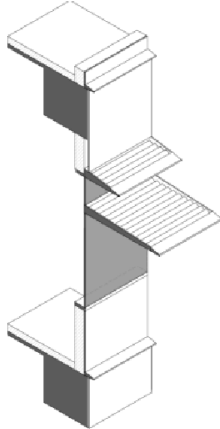

12. $1.65 \mathrm{~m}^{2} / \mathrm{m}$ 13. $1.75 \mathrm{~m}^{2} / \mathrm{m}$

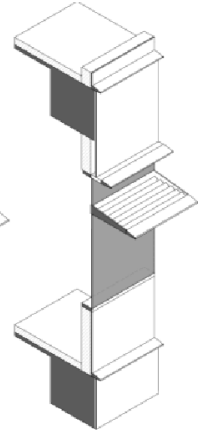

14. $1.0 \mathrm{~m}^{2} / \mathrm{m}$

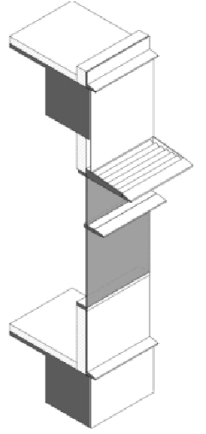

15. $1.0 \mathrm{~m}^{2} / \mathrm{m}$ FINAL CASE
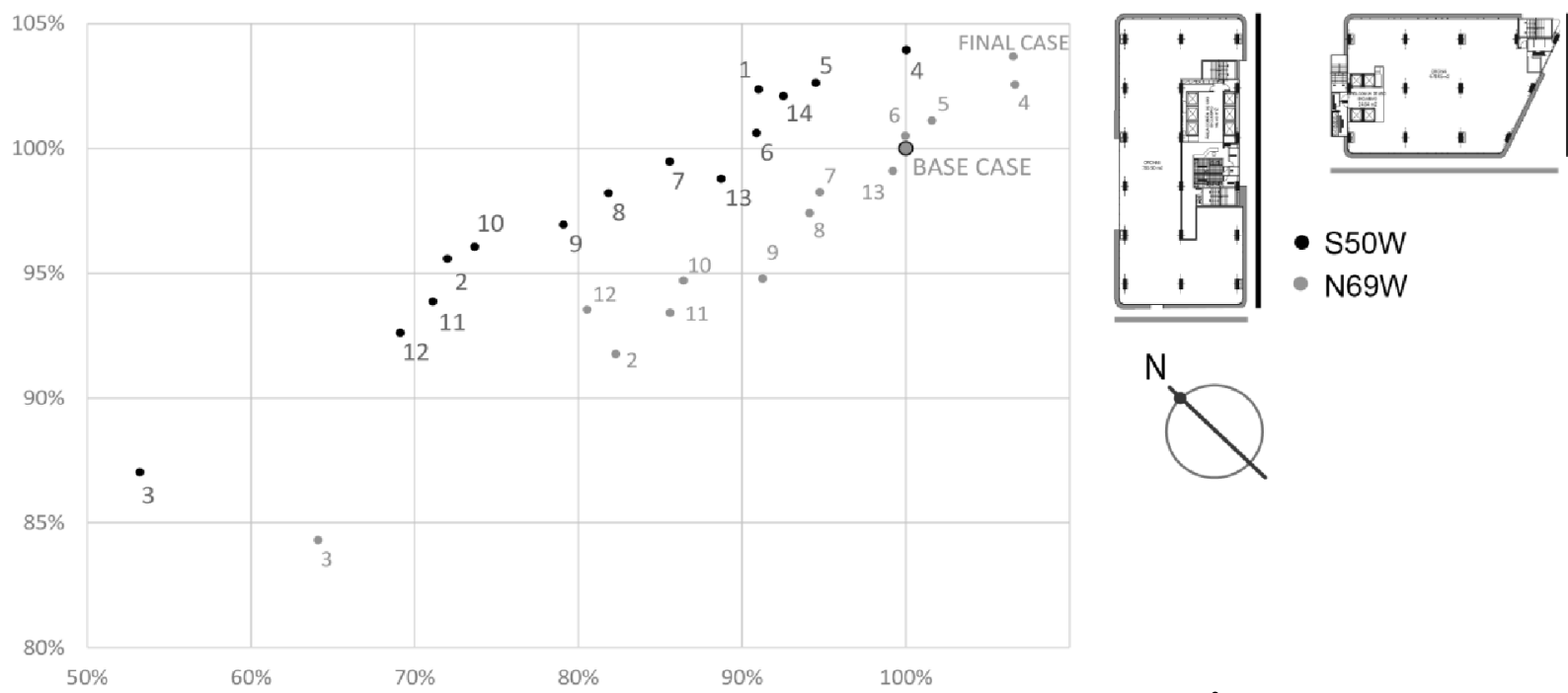

$\mathrm{N}$

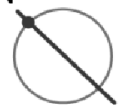

Fig. 8 Alternatives evaluated during the design phase and its aluminum requirements in $\mathbf{m}^{2}$ of aluminum per linear meter of facade.

alternatives were evaluated with two horizontal eaves with varied width and spacing (alternatives 6-11). Subtle geometric differences revealed practical changes in the solar performance achieved. From alternative 14 the engineers responsible for designing the air conditioning system began to make detailed calculations of thermal loads to finally specify the type of glass to be used without requiring changes in the already designed shade devices.

\section{Conclusions}

The Solar Coupling Index and the diagrams that represent it are easy to interpret tools that help promote high solar performance from the early stages of design. The method allows, based on basic geometric information, a quantitative comparison of the decision effectiveness aimed at minimizing unwanted solar incursion while promoting the use of natural lighting.

The method to calculate the Solar Coupling Index allows a performance threshold to be defined as a starting point of the process and then calculating its margin of optimization. The analysis resolution is sufficient to differentiate the effectiveness in solar control of shadow devices that are practically indistinguishable from the formal point of view.

The common values of Solar Coupling will often be far from the ideal value, but the possibility of quantifying this distancing with respect to a theoretical situation constitutes an impartial method to measure the gap of the solar yield of any shade device with respect to the best possible condition attained. 


\section{References}

[1] Mardaljevic, J., Heschong, L., and Lee, E. 2009. "Daylight Metrics and Energy Savings." Lighting Research \& Technology 41 (3): 261-83.

[2] Rogers, Z. 2006. "Daylighting Metric Development Using Daylight Autonomy Calculations in the Sensor Placement Optimization Tool." Architectural Energy Corporation, Boulder, Colorado, USA.

[3] Monteoliva, J. M., Villalba, A., and Pattini, A. 2012. "Impacto de la utilización de bases climáticas regionales en la simulación de alta precisión de iluminación natural." Avances en Energías Renovables y Medio Ambiente 16.

[4] Nielsen, M., Svendsen, S., and Jensen, B. L. 2011. "Quantifying the Potential of Automated Dynamic Solar Shading in Office Building through Integrated Simulations of Energy and Daylight." Solar Energy 85: 757-68.

[5] Reinhart, C., Mardaljevic, J., and Rogers, Z. 2006. "Dynamic Daylight Performance Metrics for Sustainable Building Design." LEUKOS 3 (1): 1-20.

[6] Jorge, S. 2017. "Solar Control Design by Matching Criteria between Its Shading Mask and the Shadow Desirability Schedule to Improve Natural Daylighting in an Office Building in the Tropic." In PLEA Proceedings of Passive and Low Energy Architecture Conference, Edimburg, pp. 740-7.

[7] William, O., Konstantinos, K., and Andreas, K. 2012. "Manually-Operated Window Shade Patterns in Office
Buildings: A Critical Review." Building and Environment 60: 319-38

[8] Bodart, M., and De Herde, A. 2002. "Global Energy Savings in Office Buildings by Use of Daylighting." Energy and Buildings 34 (5): 421-9.

[9] Leyla, S., and Michael, U. 2013. "The Effect of Window Shading Design on Occupant Use of Blinds and Electric Lighting." Building and Environment 64: 67-76. doi:10.1016/ j.buildenv.2013.02.013.

[10] Olgyay, V. 1963. Design with Climate: Bioclimatic Approach To Architectural Regionalism. Princeton University Press.

[11] Lippsmeier, G. 1969. Tropenbau, Building in the Tropics. Munich: Ed. Callwey Verlag.

[12] Baruch, G. 1976. Man, Climate and Architecture. London: Applied Science Publishers.

[13] Szokolay, S. V. 1977. Solar Energy and Building. London: Architectural Press.

[14] Yáñez, G. 1988. Arquitectura Solar. Madrid: MOPU.

[15] Kolivand, H., and Shahrizal Sunar, M. A. 2013. "Survey of Shadow Volume Algorithms in Computer Graphics." IETE Technical Review 30 (1): 38-46.

[16] Newman, W., and Sproull, R. 1973. Principles of Interactive Computer Graphics. New York: McGraw-Hill Book Company. p. 607. ISBN 0-07-046337-9.

[17] Salazar, J. 2009. “Técnicas del paisaje.” Presented at Encontro Latinoamericano de Conforto no Ambiente Construido, Natal, pp. 1735-44. 